\title{
ELEMENTS EVALUATION OF SOFT-HARDWARE OBJECTS WITH COMPLEX PHYSICAL, INFORMATION STRUCTURES LINKS
}

\author{
Pavel Khusainov \\ Military Institute of Telecommunications and Informatization
}

Abstract. It is offered to consider the index of element importance for multi-element soft-hardware objects with complex structure physical, information links. Elements evaluation is carried out in the planning of implementation of diagnostic tests. The methodological basis is based on the use of formal objects "network of uniformity soft-hardware devices", "processes operating environment", the diagnostic model of inter-process communication tract.

Keywords: technical diagnostics, inter-process communication, complex network, logical conclusion

\section{OCENA ELEMENTÓW OBIEKTÓW OPROGRAMOWANIA I SPRZĘTU O ZLOŻONEJ STRUKTURZE KOMUNIKACJI FIZYCZNYCH, INFORMACYJNYCH}

Streszczenie. W artykule przedstawiono analizę możliwości wykorzystania wskaźnika oceny wieloelementowych obiektów oprogramowania i sprzętu o złożonej strukturze komunikacji fizycznej i informacyjnej. Ocena elementów odbywa się przy planowaniu wykonania testów diagnostycznych. Podstawa metodologiczna oparta jest na wykorzystaniu obiektów formalnych "sieć tego samego typu oprogramowania i sprzętu", "środowisko operacyjne procesów obliczeniowych", modelu komunikacji międzyprocesowej.

Słowa kluczowe: diagnostyka techniczna, komunikacja międzyprocesowa, sieć złożona, wniosek logiczny

\section{Introduction}

Technical diagnostics soft-hardware objects with complex structure physical, information links (the object of diagnosis, OD) should be carried out taking into account the systemic role of their elements. The methodological basis of technical systems' analysis with a sequential, sequential-parallel, parallel-sequential structure of elements is sufficiently developed and tested. At the same time, evaluation of elements soft-hardware objects with a more complex structural organization requires further research.

A perspective approach to evaluating of the structural properties of elements of multi-element soft-hardware objects is their consideration as elements of the cybernetic system $[3,10]$.

Cybernetic system is an abstract model of a technical system as a set of related objects (elements) capable to receive, store, process, and exchange information. The scientific research of cybernetic systems is aimed to determine the most common patterns for all such objects $[5,8]$ :

- structure - a set of elements and relations between them;

- state - a set of states of elements and connections between them;

- the complexity is due to the dimensional (the number of parameters which describe elements' states), number and variety of relationships between elements.

The presentation of OD as a set of physical, informational complex structures of links cybernetic system connections allows us to use a wide range of interpretations for complex networks. The theory of complex networks offers the most developed approach to description and evaluation of the structural properties of multi-element technical objects [2,4].

\section{Formulation of the problem}

Based on the foregoing, the model of the selected OD is the formal object "network of uniformity soft-hardware devices". The assumption of "uniformity" is accepted on the basis of analysis of trends, principles of practical implementation (modularity, multilevel, unification) software, hardware support of Open System Interconnection (OSI) and inter-process communication.

It is offered to evaluate the element importance to support physical, informational connectivity of multi-element softwarehardware ODs. The multipliers of additive index $E_{i}^{\chi}$ are the relative weights of element inclusion in the structures of physical, informational connectivity on the observation interval $\chi$. The initial data are the adjacency matrix of the formal object "network of uniformity soft-hardware devices" in the observation interval $\chi$ : for the graph of physical links $\left\|u_{i j}^{\chi}\right\|$, for the graph of information links $\left\|w_{i j}^{\chi}\right\|$.

\section{The network of uniformity soft-hardware devices}

The network of uniformity soft-hardware devices is a formal object based on a one-to-one mapping of physical and information connectivity of OD elements to the set of nodes complex network and relationships between them (Fig. 1):

$$
M=<V, U, W, T>\text {. }
$$

Notation:

$V=\left\{v_{i}\right\}$ is a set of uniformity soft-hardware devices;

$U$ is a relation of physical connectivity;

$W$ is a relation of informational connectivity;

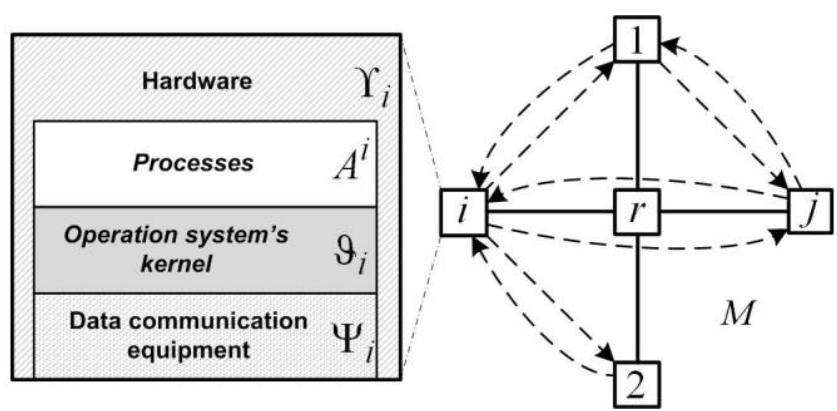

Fig. 1. Example of a formal object "network of uniformity soft-hardware devices"

$T=\left\{t_{s}\right\}$ is a set of discrete values of registration time of OD's states, $s \in Z_{0}^{+}, \quad T=\bigcup_{\chi=1}^{|\mathrm{X}|} T^{\chi}, \quad \bigcap_{\chi=1}^{|\mathrm{X}|} T^{\chi}=\varnothing, \quad \mathrm{X}$ is a set of time intervals for monitoring the $\mathrm{OD}, T^{\chi}=\left\{t_{s \chi}\right\}$ is a set of discrete time values of registration OD's states on the observation interval.

The composition of uniformity soft-hardware device [6, 7]:

- hardware (CPU, RAM, system bus, timer, etc.);

- data communication equipment, DCE (I/O device);

- kernel of operating system of soft-hardware device;

- processes. 
A process is a unit for planning computing work of a softhardware device and the subject of access to resources. The algorithm of process software implementation in RAM determines the computational work of soft-hardware device on the time interval for its execution.

The basis for building a process of software implementation in RAM is the loadable module of a program. The memory address space required for the process is isolated from unforeseen I/O (Input/Output) operations.

The operating system kernel is a software implementation of control algorithms for scheduling and allocation of CPU time, address space RAM, I/O devices between processes, providing their access to system services. The role and place of the kernel of operating system is to provide inter-process communication in the context of the I/O operations. Its software implementation is provided by functionality of OSI levels: transport, network, data link. For this, its software implementation includes: firstly, the functionality of the transport, network, data link layer; secondly, the provision of FIFO service discipline, stream multiplexing, error control, etc.

Vectors of parameter values and settings of functional components of a uniformity soft-hardware device, $v_{i} \in V$, $i=\overline{1,|V|}$ (Fig. 1, 2):

$$
v_{i}=<\Upsilon_{i}, \Psi_{i}, \vartheta_{i}, A^{i}>
$$

Notation:

$\Upsilon_{i} \in \Upsilon, \Upsilon=\left\{U_{\alpha}\right\}$ is a set of variants of vector fixed values $v_{\alpha}=(\Xi)$ where $\Xi=\left\{\xi_{\zeta}\right\}$ is a set of parameters and settings of hardware components, $\zeta=\overline{1,|\Xi|}, \alpha=\overline{1,|\Upsilon|}$;

$\Psi_{i} \in \Psi, \quad \Psi=\left\{\psi_{\varsigma}\right\} \quad$ is a set of variants of vector fixed values of parameters and settings DCE, $\varsigma=\overline{1,|\Psi|}$, $\left.\psi_{\zeta}=\left(H^{i} \times K \times \mathrm{B}^{k}\right)=\left(<h_{i}, k, \beta^{k}\right\rangle\right), \quad h_{i}=\overline{1,\left|H^{i}\right|}, \quad K$ is a types of DCE device, $k=\overline{1,|K|}, \quad \mathrm{B}^{k}=\left\{\beta_{\mathrm{w}}^{k}\right\} \quad$ is a set of parameters and settings of DCE device components, $\omega=\overline{1,\left|\mathrm{~B}^{k}\right|}$;

$\vartheta_{i} \in \vartheta, \vartheta=\left\{\varphi_{\mathrm{o}}\right\}$ is a set of options and settings of software implementation kernel components to provide inter-process communication, $\quad \varphi_{\mathrm{o}}=<f_{x}, f_{d}, f_{y}, f_{z}, f_{l}>, \quad \mathrm{o}=\overline{1,|\vartheta|}, \quad f_{x}^{i} \in F(X)$, $f_{d}^{i} \in F(D), f_{y}^{i} \in F(Y), f_{z}^{i} \in F(Z), f_{l}^{i} \in F(L), x=\overline{1,|F(X)|}$, $d=\overline{1,|F(D)|}, \quad y=\overline{1,|F(Y)|}, \quad z=\overline{1,|F(Z)|}, \quad l=\overline{1,|F(L)|}$, where $F(X), \quad F(D), \quad F(Y), \quad F(Z), \quad F(L)$ is a set of options and settings of software implementation typical kernel services to provide inter-process communication:

$f_{x}^{i}$ - access to $\mathrm{I} / \mathrm{O}$ buffers of the kernel when executing of processes software implementations $A^{i}$ in the RAM;

$f_{d}^{i}$ - buffering of blocks of data when executing I/O call of the kernel of process software implementations $A^{i}$ in the RAM;

$f_{y}^{i}$ - multiplexing (demultiplexing) I/O streams of the kernel;

$f_{z}^{i}$-dispatching I/O streams of the kernel;

$f_{l}^{i}$ - buffering I/O data blocks of the DCE.

The role and place of kernel components of soft-hardware device are considered in the context of formal object "processes operating environment" (Fig. 2):

$$
v_{i}=<A^{i}, O^{i}, G^{i}, f_{x}^{i}, f_{d}^{i}, f_{y}^{i}, f_{z}^{i}, f_{l}^{i}>,
$$

where $A^{i}=\left\{a_{n b}^{i}\right\}$ is a set of processes software implementations in the RAM, $n=\overline{1,\left|A^{i}\right|}, b=\overline{1,|F(B)|}, F(B)$ is a set of variants of processes software implementation and setting its algorithm in the RAM $a_{n b}^{i} \in A^{i}$ created on the basis of programs loading module $b ; O^{i}=\left\{o_{e}^{i}\right\}$ is a set of kernel I/O buffers, $e=\overline{1,\left|O^{i}\right|}$; $G^{i}=\left\{g_{h k}^{i}\right\}$ is a set of DCE I/O buffers, $h_{i}=\overline{1,\left|H^{i}\right|}, k=\overline{1,|K|}$.

Access to the kernel I/O buffers when executing processes software implementations on the observation interval $\chi$ is determined by the truth of the predicate $X^{\chi}\left(a_{n b}^{i}, o_{e}^{i}\right)$ and is provided by the operability $f_{x}^{i}, f_{d}^{i}$ :

$$
\begin{gathered}
X^{\chi}\left(a_{n b}^{i}, o_{e}^{i}\right)=A^{i} \times O^{i}=\left\|x_{i n e}^{\chi}\right\|=\left\{\begin{array}{l}
1, T R U E, \\
0, F A L S E,
\end{array}\right. \\
f_{x}^{i}: a_{n b}^{i} \cdots \cdots o_{e}^{i}\left[f_{d}^{i}\right],
\end{gathered}
$$

where $\left\|x_{\text {ine }}^{\chi}\right\|$ is access matrix for transferring data blocks between memory address spaces of process $a_{n b}^{i} \in A^{i}$ and kernel I/O buffers $o_{e}^{i} \in O^{i}$

Multiplexing (demultiplexing) of kernel I/O streams on the observation interval $\chi$ is determined by the truth of the predicate $Y^{\chi}\left(o_{e}^{i}, g_{h k}^{i}\right)$ and is provided by the operability $f_{d}^{i}, f_{y}^{i}, f_{l}^{i}$ :

$$
\begin{gathered}
Y^{\chi}\left(o_{e}^{i}, g_{h k}^{i}\right)=O^{i} \times G^{i}=\left\|y_{i e h}^{\chi}\right\|=\left\{\begin{array}{l}
1, \text { TRUE }, \\
0, F A L S E,
\end{array}\right. \\
f_{y}^{i}: o_{e}^{i}\left[f_{d}^{i}\right] \cdots \cdots g_{h k}^{i}\left[f_{l}^{i}\right],
\end{gathered}
$$

where $\left\|y_{i e h}^{\chi}\right\|$ is the adjacency matrix of directions of transfer graph of data blocks between memory address spaces of kernel I/O buffers $o_{e}^{i} \in O^{i}$ and DCE I/O buffers $g_{h k}^{i} \in G^{i}$.

Dispatching of kernel I/O streams on the observation interval $\chi$ is determined by the truth of the predicate $Z^{\chi}\left(o_{e}^{i}, g_{h k}^{i}\right)$ and is provided by the operability $f_{d}^{i}, f_{y}^{i}, f_{z}^{i}, f_{l}^{i}$ :

$$
\begin{gathered}
Z^{\chi}\left(o_{e}^{i}, g_{h k}^{i}\right)=\left\|y_{i e h}^{\chi}\right\| \cdot\left\|z_{i e h}^{\chi}\right\|=\left\{\begin{array}{c}
1, \text { TRUE, } \\
0, \text { FALSE, }
\end{array},\right. \\
f_{z}^{i}: o_{e}^{i}\left[f_{d}^{i}\right] \cdots \cdots \cdot\left\|z_{\text {eh }}^{i}\right\| \cdots \cdots \cdot g_{h k}^{i}\left[f_{l}^{i}\right],
\end{gathered}
$$

where $\left\|x_{i n e}^{\chi}\right\|$ is access matrix for transferring data blocks of kernel I/O streams between memory address spaces of kernel I/O buffers $o_{e}^{i} \in O^{i}$ and DCE I/O buffers $g_{h k}^{i} \in G^{i}$.

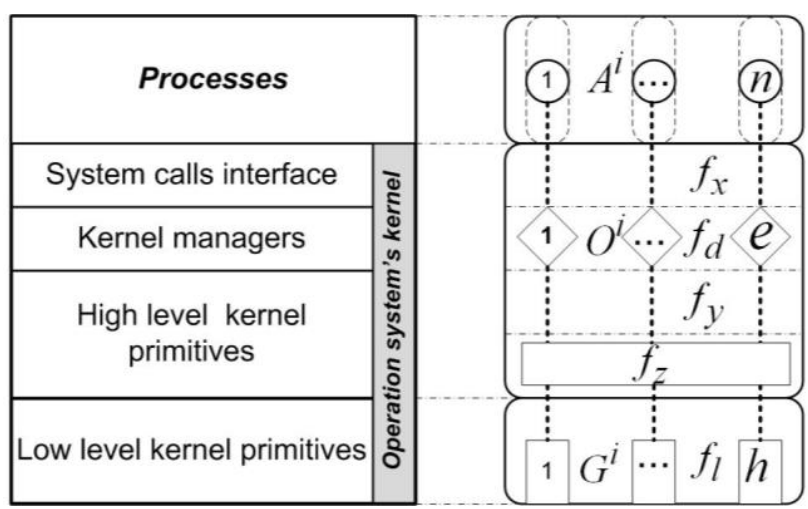

Fig. 2. A formal object processes operating environment 
Principles to evaluate the element importance of OD on the basis of a formal object "network of uniformity softhardware devices":

- the relation of nodes' physical connectivity $U$ is mapping of operability physical connection between OD's elements (data transmission channel);

- the relation of nodes' information connectivity $W$ is mapping of operability information links between processes OD's elements (inter-process communication tract);

- the graphs of physical, information links between elements of OD are mapping of technical state of inter-process communication tracts and data transmission channels in their composition.

Predicates of physical connectivity $v_{i}, v_{j} \in V$ on the observation interval $\chi$ :

$$
\begin{gathered}
U^{\chi}\left(v_{i}, v_{j}\right)=V \times V=\left\|u_{i j}^{\chi}\right\|=\left\{\begin{array}{l}
1, \text { TRUE }, \\
0, F A L S E,
\end{array}\right. \\
U^{\chi}\left(h_{i}, h_{j}\right)=H \times H=\left\|u_{i j}^{\chi}\right\|=\left\{\begin{array}{l}
1, T R U E, \\
0, F A L S E,
\end{array}\right. \\
U^{\chi}\left(v_{i}, v_{j}\right) \equiv U^{\chi}\left(h_{i}, h_{j}\right),
\end{gathered}
$$

where $\left\|u_{i j}^{\chi}\right\|$ is the relationship matrix of nodes physical connectivity for formal object "network of uniformity softhardware devices" is an equivalent to the adjacency matrix of graph of physical connections between the elements of OD $M_{U}^{\chi}(V)$ on the observation interval $\chi$ (data transmission channels), $\chi=\overline{1,|\mathrm{X}|}, H=H^{i} \cup H^{j}, H^{i} \cap H^{j}=\varnothing, h_{i}=\overline{1,\left|H^{i}\right|}$, $h_{j}=\overline{1,\left|H^{j}\right|}, i, j=\overline{1,|V|}, i \neq j$.

Predicates of information connectivity $v_{i}, v_{j} \in V$ on the observation interval $\chi$ :

$$
\begin{gathered}
W^{\chi}\left(v_{i}, v_{j}\right)=V \times V=\left\|w_{i j}^{\chi}\right\|=\left\{\begin{array}{l}
1, \text { TRUE }, \\
0, \text { FALSE, }
\end{array}\right. \\
W^{\chi}\left(a_{n b}^{i}, a_{n b}^{j}\right)=A^{i} \times A^{j}=\left\|w_{i j}^{\chi}\right\|=\left\{\begin{array}{l}
1, T R U E, \\
0, F A L S E,
\end{array}\right. \\
W^{\chi}\left(v_{i}, v_{j}\right) \equiv W^{\chi}\left(a_{n b}^{i}, a_{n b}^{j}\right),
\end{gathered}
$$

where $\left\|w_{i j}^{\chi}\right\|$ is the relationship matrix of nodes information connectivity for formal object "the network of uniformity softhardware devices" is an equivalent to the adjacency matrix of graph of information links between OD's elements $M_{W}^{\chi}(V)$ on the observation interval $\chi$ (inter-process communication tracts), $\chi=\overline{1,|\mathrm{X}|}, i, j=\overline{1,|V|}, i \neq j$.

Recall $[1,4,9]$ that a graph is a set of nodes (vertices) and their ordered (unordered) pairs. An ordered pair of nodes (vertices) is called an edge; an unordered pair is called an arc. A pair of nodes (vertices) can be connected by two or more edges (arcs). Common graphs naming in applied research and engineering tasks is complex network. The element of the graph adjacency matrix is a mapping of links number between nodes, the element of the graph incidence matrix - nodes which are incidental to links. The graph node degree is mapping of edges number that is incidental to the node of the graph. The sum of the graph edges is equal to the sum of the degrees of its nodes.

Two nodes of the graph are called connected if there is a route between them. A route is a finite sequence edges to move from one node to another. Cartesian product of sets is mapping binary relation of elements connectivity and can be given in terms of the predicate calculus. A binary relation is any subset of ordered pairs of Cartesian product.
The truth of the predicate indicates the existence of a relationship. The truth of the predicate indicates the validity of a binary relation connectivity as a result of the Cartesian product.

To define a relation means to set a predicate (method) of selecting a subset of ordered pairs from the set of results of Cartesian product. A visual representation of binary relations is the relation matrix, an oriented (undirected) graph or its adjacency matrix.

\section{The formal axiomatic apparatus for determining the technical state of inter-process communication tract}

The conclusion about the technical state of the inter-process communication tract is equivalent to the truth's conjunction of results of diagnostic tests of its functional elements (Fig. 3):

$$
\begin{gathered}
\tilde{W}^{\chi}\left(a_{n b}^{i}, a_{n b}^{j}\right) \Leftrightarrow R\left(w_{i j}^{\chi}\right), \\
R\left(v_{i}\right) \wedge R\left(u_{i r}^{\chi}\right) \wedge R\left(v_{r}\right) \wedge R\left(u_{r j}^{\chi}\right) \wedge R\left(v_{j}\right) \supset R\left(w_{i j}^{\chi}\right), \\
R\left(f_{x}^{i}\right) \wedge R\left(f_{d}^{i}\right) \wedge R\left(f_{y}^{i}\right) \wedge R\left(f_{z}^{i}\right) \wedge R\left(f_{l}^{i}\right) \supset R\left(v_{i}\right), \\
R\left(f_{x}^{j}\right) \wedge R\left(f_{d}^{j}\right) \wedge R\left(f_{y}^{j}\right) \wedge R\left(f_{z}^{j}\right) \wedge R\left(f_{l}^{j}\right) \supset R\left(v_{i}\right), \\
R\left(f_{l_{i}}^{r}\right) \wedge R\left(f_{z}^{r}\right) \wedge R\left(f_{l_{j}}^{r}\right) \supset R\left(v_{r}\right) .
\end{gathered}
$$

Notation:

$R\left(v_{i}\right), R\left(v_{r}\right), R\left(v_{j}\right)$ are indicators of results diagnostic tests that is mapping of technical state of the processes operating environment (2-9), $v_{i}, v_{j}, v_{r} \in V, i, j, r=\overline{1,|V|}, i \neq j \neq r$;

$R\left(u_{i r}^{\chi}\right), R\left(u_{r j}^{\chi}\right)$ are indicators of results diagnostic tests that is mapping of technical state of the data transmission channels;

$R\left(f_{x}^{i(j)}\right), \quad R\left(f_{d}^{i(j)}\right), \quad R\left(f_{y}^{i(j)}\right), \quad R\left(f_{z}^{i(j)}\right), \quad R\left(f_{l}^{i(j)}\right)$ are an indicators of results diagnostic tests that is mapping of typical kernel services to provide inter-process communication of the terminal soft-hardware device;

$R\left(f_{l_{i}}^{r}\right), R\left(f_{z}^{r}\right), R\left(f_{l_{j}}^{r}\right)$ are indicators of results diagnostic tests

is mapping of typical kernel services to provide inter-process communication of the transit soft-hardware device.

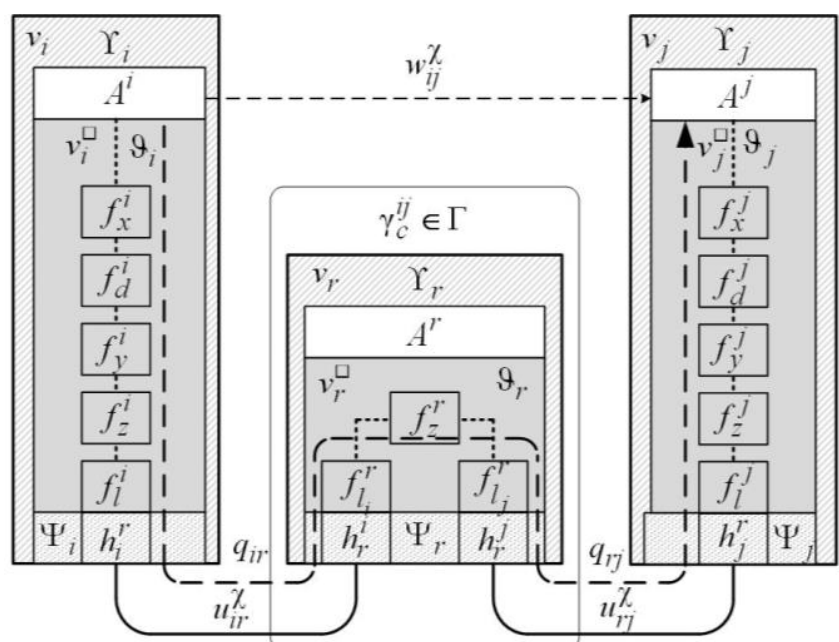

Fig. 3. A inter-process communication tract

The conclusion about the technical state of data transmission channels is equivalent to the truth conjunction of results diagnostic tests of its DCE and physical transmission medium, $i, j, r=\overline{1,|V|}$, $i \neq j \neq r, \chi=\overline{1,|\mathrm{X}|}$ (Fig. 3):

$$
\tilde{U}^{\chi}\left(h_{i}, h_{j}\right) \Leftrightarrow R\left(u_{i j}^{\chi}\right),
$$




$$
\begin{aligned}
& R\left(h_{i}^{r}\right) \wedge R\left(q_{i r}\right) \wedge R\left(h_{r}^{i}\right) \supset R\left(u_{i r}^{\chi}\right), \\
& R\left(h_{r}^{j}\right) \wedge R\left(q_{r j}\right) \wedge R\left(h_{j}^{r}\right) \supset R\left(u_{r j}^{\chi}\right) .
\end{aligned}
$$

Notation:

$R\left(h_{i}^{r}\right), R\left(h_{r}^{i}\right), R\left(h_{r}^{j}\right), R\left(h_{j}^{r}\right)$ are indicators of results diagnostic tests that is mapping of technical state of the DCE;

$R\left(q_{i r}\right), R\left(q_{r j}\right)$ are indicators of results diagnostic tests that is mapping of technical state of physical transmission medium.

The methodological basis of formal axiomatic apparatus (16-23) is the calculus of statements, the predicate logic using a theory of cybernetic systems, logical models of diagnostic. Based on the values $(16,21)$, the adjacency matrices of the physical connections between elements of OD $\left\|u_{i j}^{\chi}\right\|$, information links between elements of OD $\left\|w_{i j}^{\chi}\right\|$ are formed.

\section{The index of the element OD's importance}

Based on the foregoing, on (10-15) as an index of the element importance of OD $E_{i}^{\chi}$ on the observation interval $\chi$ it is proposed, $i=\overline{1,|V|}, v_{i} \in V$ :

$$
\begin{gathered}
E_{i}^{\chi}=\frac{\aleph_{i}^{\chi}(U)}{\sum_{i=1}^{|V|} \aleph_{i}^{\chi}(U)} \cdot \frac{\aleph_{i}^{\chi}(W)}{\sum_{i=1}^{|V|} \aleph_{i}^{\chi}(W)}, \\
\aleph_{i}^{\chi}(U)=\sum_{j=1}^{|V|-1} u_{i j}^{\chi}, \\
\aleph_{i}^{\chi}(W)=\sum_{j=1}^{|V|-1} w_{i j}^{\chi} .
\end{gathered}
$$

where $\boldsymbol{\aleph}_{i}^{\chi}(U)$ is a node degree of physical connectivity for formal object "network of uniformity soft-hardware devices" which is equivalent to the degree element OD of physical connections; $\aleph_{i}^{\chi}(W)$ is a node degree of information connectivity for formal object "network of uniformity soft-hardware devices" which is equivalent degree element OD of information links. At the same time, the degree of nodes information connectivity which is a mapping of transit elements OD $v_{r} \in V$ is defined as the sum of inter-process communication tracts between $v_{i}, v_{j} \in V$ :

$$
\boldsymbol{\aleph}_{r}^{\chi}(W)=\sum_{i=1}^{|V|} \sum_{j=1}^{|V|-1} w_{i j r}^{\chi} .
$$

Consider an example of assessing the OD's elements importance as nodes of a formal object "network of uniformity soft-hardware devices" and appropriate diagnostic model (Fig. 4).
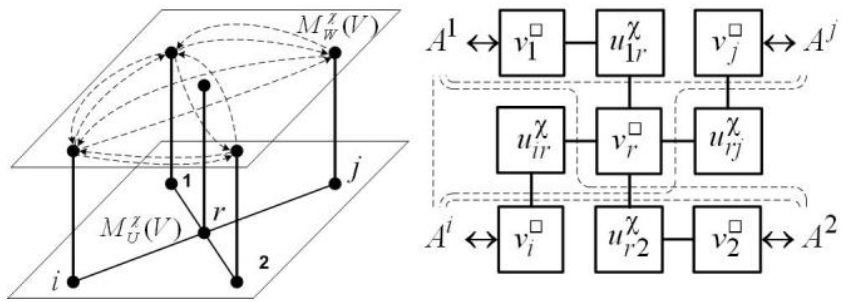

Fig. 4. An example of network of uniformity soft-hardware devices
Analysis of adjacency matrix of graphs of physical $M_{U}^{\chi}(V)$, information $M_{W}^{\chi}(V)$ connectivity revealed such values of the degrees of its nodes on the observation interval $\chi$ : $\aleph_{1}^{\chi}(U), \aleph_{i}^{\chi}(U), \aleph_{j}^{\chi}(U), \aleph_{2}^{\chi}(U)=1, \quad \aleph_{r}^{\chi}(U)=4, \quad \aleph_{j}^{\chi}(W)=2$, $\boldsymbol{\aleph}_{r}^{\chi}(W)=8, \boldsymbol{\aleph}_{i}^{\chi}(W)=6, \boldsymbol{\aleph}_{1}^{\chi}(W), \boldsymbol{\aleph}_{j}^{\chi}(W)=4$.

Based on the foregoing, index of the element OD importance is $E_{1}^{\chi} \approx 0,02, E_{i}^{\chi} \approx 0,03, \quad E_{r}^{\chi} \approx 0,17, E_{j}^{\chi} \approx 0,02, E_{2}^{\chi} \approx 0,01$.

The prevailing index of the element OD's importance $\left(E_{r}^{\chi} \approx 0,17\right.$ ) is explained by the obvious transit role of this OD's element. The main element of the tracts inter-process communication is the functional component $v_{r} \in V$ described by the formal object "processes operating environment".

\section{Conclusions}

Adequacy of the element OD importance index (24-25) is based on generally accepted interpretation of a node degree of a graph (complex network), it is informative, invariant, and resistant to evaluation interference.

Formal objects "network of uniformity soft-hardware devices" (1-2, 10-15), "process's operating environment" (3-9) are the basis of a new scientific approach to the synthesis and analysis of multi-element soft-hardware objects with a complex structure of physical, information links. The formal axiomatic apparatus (16-23) is a logical conclusion system about the technical state of an inter-process communication tracts. The conclusion about the technical state of "network of uniformity soft-hardware devices" can be made after performing diagnostic tests based on a comparison of their results using index of the element OD importance (24-25).

Further research is aimed to develop a model of diagnostic parameter of inter-process communication tracts and methods of evaluation of technical state network of uniformity softhardware devices.

\section{References}

[1] Gerasimov B. M., Eydel'man S. D., Sirchenko Z F., Shuman B. M Matematicheskiye osnovy issledovaniya operatsiy (I). Diskretnyye struktury: Uchebnoye posobiye. KVIRTU PVO, Kiev 1989.

[2] Holovach YU.: Skladni merezhi. Zhurnal fizychnykh doslidzhen' (t. 10), 4/2006, 247-289.

[3] Klyuyeva V. V., Parkhomenko P. P. (red.): Nadezhnost' i effektivnost' v tekhnike: Spravochnik (10 t). Mashinostroyeniye, Moscow 1987

[4] Ore O.: Teoriya grafov. Nauka, Moscow 1980.

[5] Slovar' po kibernetike. USE im. M.P. Bazhana, Kiev 1989.

[6] Tanenbaum E.: Sovremennyye operatsionnyye sistemy. Piter, Sankt Petersburg 2012.

[7] Uaynder S.: Spravochnik po tekhnologiyam i sredstvam svyazi. Mir, Moscow 2000.

[8] Vinogradov I. M. (red.): Matematicheskaya entsiklopediya. Sovetskaya Entsiklopediya (t. 4), Moscow 1984

[9] Vinogradov I.M. (red.): Matematicheskaya entsiklopediya (t. 1). Sovetskaya entsiklopediya, Moscow 1977.

[10] Zherdyev M. K., Lyenkov S. V., Kredentser B. P. ta in.: Fizychni osnovy teoriy nadiynosti: pidruchnyk. Kyyivs'kyy universytet, Kiev 2008.

\section{Ph.D. Pavel Khusainov}

e-mail: indesys@ukr.net

Began a scientific career in 2004. In 2007 she was awarded with the degree of Ph.D. (technical sciences). Held position Associate Professor, Military Institute of Telecommunication and Informatization, Kyiv. Pedagogical experience more than of 10 years.

Scientific activities: technical diagnostics, cybersecurity of complex multi-element soft-hardware systems. Author (coauthor) of more than 60 scientific papers and methodical publications.

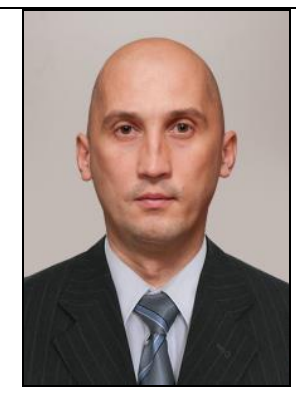

ORCID ID: 0000-0002-0675-0369

otrzymano/received: 01.12 .2018 przyjęto do druku/accepted: 28.02 .2019 